\title{
Trombosis venosas cerebrales en la edad pediátrica: presentación clínica, factores de riesgo, diagnóstico y tratamiento
}

\author{
M. Eugenia Russi, Verónica González, Jaume Campistol
}

Introducción. Desde su primera descripción a mediados del siglo XIX, mucho se ha avanzado en el conocimiento de esta entidad, que constituye una reconocida, aunque desestimada, causa de ictus en la infancia.

Objetivo. Analizar la presentación clínica, factores de riesgo, evolución y tratamiento de una población de niños con trombosis venosa cerebral.

Pacientes y métodos. Se llevó a cabo un estudio descriptivo, retrospectivo y longitudinal, de niños entre 0 y 17 años con trombosis venosa cerebral, en un período de cinco años.

Resultados. Se identificaron 31 casos, de los cuales 18 fueron varones. Dieciocho pacientes comenzaron con convulsiones y 13 con síndrome de hipertensión endocraneal. Se identificaron 23 pacientes con procesos infecciosos, cuatro con patología de base previa, cinco con factores protrombóticos, tres neonatos con traumatismo obstétrico, cinco con más de un factor de riesgo y uno sin marcadores de riesgo. Se realizó diagnóstico por resonancia magnética en 18 pacientes, por tomografía computarizada con contraste en 11 y por angiorresonancia en los dos restantes. Dieciséis presentaron trombosis del seno transverso, cuatro del longitudinal superior, cuatro del sigmoides, cinco afectación de más de un seno y dos del seno cavernoso. Se inició anticoagulación en 21 niños y no se trataron los 10 restantes. Doce pacientes manifestaron déficit neurológico a largo plazo, mientras que 18 se mantuvieron asintomáticos. No hubo fallecimientos o complicaciones graves por la trombosis ni la anticoagulación, así como tampoco recurrencias sintomáticas en el seguimiento.

Conclusiones. Las trombosis venosas cerebrales son una causa importante de ictus en la infancia. La sospecha clínica debe ser elevada ante todo paciente con clínica de hipertensión endocraneal o convulsiones en presencia de factores de riesgo conocidos.

Palabras clave. Anticoagulación. Factores de riesgo. Ictus. Infancia. Mastoiditis. Trombosis venosa cerebral.

\section{Introducción}

Consideradas desde mediados del siglo xix como poco frecuentes y de pronóstico reservado, las trombosis venosas cerebrales (TVC) han emergido como una reconocida causa de ictus en la infancia gracias a la implantación de modernas técnicas de neuroimagen, en especial la resonancia magnética (RM).

Con una incidencia anual estimada de 0,67 casos/100.000, está considerada como una de las causas de accidente cerebrovascular de mayor polimorfismo clínico, con una amplia gama de factores de riesgo que pueden favorecer su aparición y una respuesta generalmente favorable en la mayoría de los pacientes en los que se instaura un tratamiento adecuado y precoz $[1,2]$.

Sin lugar a dudas, su mayor reconocimiento, propiciado por el avance tecnológico experimentado en las últimas décadas en el campo de la neuroimagen, ha despertando una creciente preocupación por la que aún hoy día continúa siendo una patología percibida como excepcional.

El principal objetivo de este trabajo se centra en la descripción de una población de niños con TVC, analizando su presentación clínica, factores de riesgo, diagnóstico, pronóstico y tratamiento.

\section{Pacientes y métodos}

Se llevó a cabo un trabajo descriptivo, retrospectivo y longitudinal de niños de entre 0 (con una edad gestacional mayor de 36 semanas) y 17 años, ingresados en el Hospital Sant Joan de Déu (Barcelona), durante el período comprendido entre 2004 y 2009, con diagnóstico de TVC. Para ello se elaboró un
Servicio de Neuropediatría. Hospital Sant Joan de Déu. Esplugues de Llobregat, Barcelona, España.

Correspondencia:

Dra. María Eugenia Russi. Servicio de Neurología. Hospital Sant Joan de Déu. Pg. Sant Joan de Déu, 2. E-08950 Esplugues de Llobregat (Barcelona).

Fax:

+34932033959.

E-mail:

mrussi@hsjdbcn.es

Aceptado tras revisión externa: 05.10.10.

Cómo citar este artículo: Russi ME, González V, Campistol J. Trombosis venosas cerebrales en la edad pediátrica: presentación clínica, factores de riesgo, diagnóstico y tratamiento. Rev Neurol 2010; 51: 661-8.

C 2010 Revista de Neurología 
protocolo de recogida de datos que incluía edad, sexo, antecedentes personales y familiares, presentación clínica, factores de riesgo, estudios complementarios, tratamiento y evolución. Se dividió la cohorte de estudio en tres grupos de interés: recién nacidos (0-30 días), lactantes (1 mes-1 año) y niños mayores (1-17 años). Se incluyeron todos aquellos pacientes con diagnóstico por imagen inequívoco de trombosis de senos venosos cerebrales realizado por neurorradiólogo. Se investigó exhaustivamente la presencia de enfermedades cardíacas, renales, hematológicas, del tejido conectivo y autoinmunes, así como la existencia de factores protrombóticos: proteínas $\mathrm{C}$ y $\mathrm{S}$, antitrombina III, plasminógeno, cofactor II, protrombina 20210, factor V de Leyden, homocigosis para la variante termolábil de la metiltetrahidrofolato-reductasa (MTHFR), factor VIII, anticuerpos anticardiolipina, antifosfolípidos y anticoagulante lúpico.

Se realizó seguimiento clínico al alta. El grado de discapacidad se clasificó según la escala funcional de Rankin modificada en siete grados [3]:

\section{Asintomático.}

1. Incapacidad no significativa. Pese a la existencia de síntomas, es capaz de realizar sus tareas habituales.

2. Incapacidad leve. No puede realizar todas sus actividades previas. Realiza sus necesidades personales sin ayuda.

3. Incapacidad moderada. Requiere algún grado de ayuda, pero camina solo.

4. Incapacidad moderada-grave. Requiere ayuda en actividades diarias. No camina solo.

5. Incapacidad grave. Limitado a la cama, incontinencia, requiere cuidados de enfermería o atención constante.

6. Muerte.

\section{Resultados}

\section{Muestra}

Se incluyó en el trabajo un total de 31 pacientes $(n=31)$, de los cuales 18 eran varones (relación niño/niña 1,4 a 1 ), con un rango de edades comprendido entre 0 y 17 años, y una media de edad de 3,2 años. Seis pacientes eran neonatos, cinco lactantes y 20 niños mayores de un año. El período de seguimiento clínico fue de tres meses a cinco años, con una media de 20 meses. El tiempo medio desde el inicio de los síntomas hasta el diagnóstico fue de 6,5 días.

\section{Factores precipitantes y de riesgo}

Se identificaron factores precipitantes y de riesgo en todos menos en un paciente. Procesos infecciosos como otitis, mastoiditis, neumonía y meningitis fueron los principales factores precipitantes hallados en 23 casos (77\%), incluidos dos neonatos con sospecha de infección connatal. Se buscaron en todos ellos factores de riesgo asociados (factores protrombóticos, traumatismos o enfermedades hematooncológicas), encontrándose el factor $\mathrm{V}$ de Leyden en dos pacientes, la mutación de la MTHFR en homocigosis en otro y la mutación en heterocigosis del gen de la protrombina G20210A en dos casos. La causa traumática se atribuyó a tres neonatos cuyos partos fueron distócicos e instrumentados. Tres pacientes eran portadores de enfermedades hematooncológicas en tratamiento con quimioterapia, y un cuarto, del síndrome de Chediak-Higashi (Tabla).

\section{Presentación clínica}

Dieciocho pacientes presentaron crisis convulsivas (58\%) y los 13 restantes (42\%) signos y síntomas de hipertensión endocraneal. Dentro de este último grupo, los síntomas más constantes fueron la cefalea en nueve casos y los vómitos en otros nueve (69\%), seguidos de signos de focalidad neurológica en siete (53\%), trastornos de conciencia en cinco (38\%), irritabilidad en otros cinco (38\%), y papiledema en cuatro (30\%). No se encontraron casos asintomáticos en la serie analizada.

\section{Hallazgos radiológicos}

Se realizó diagnóstico por RM en 18 pacientes (el $58 \%$ de los casos), de los cuales 10 requirieron secuencias en fase venosa para su confirmación diagnóstica; por tomografía computarizada con contraste en 11 (36\%); y por angiorresonancia en los dos restantes (6\%). El sistema venoso superficial se vio afectado en 24 pacientes (77\%), el profundo en dos (6\%), con una afección de ambos sistemas venosos en los cinco pacientes restantes (16\%). Dentro del sistema venoso superficial, el seno más frecuentemente comprometido fue el seno transverso en 16 casos (52\%) seguido del longitudinal superior en cuatro (13\%) y del sigmoides en otros cuatro (13\%). En cuanto al sistema venoso profundo, se objetivó afectación del seno cavernoso en dos casos (6\%). Del total de pacientes, nueve presentaron imágenes hemorrágicas en la RM (29\%), tres de ellos en combinación con isquemia (9\%), y sólo dos mostraron en forma aislada imágenes isquémicas (6\%). 
Tabla. Muestra de los pacientes incluidos en el trabajo.

\begin{tabular}{|c|c|c|c|c|c|c|c|c|c|}
\hline $\begin{array}{l}\text { Paciente } \\
\text { n.o }\end{array}$ & Edad & Sexo & $\begin{array}{l}\text { Presentación } \\
\text { clínica }\end{array}$ & $\begin{array}{c}\text { Factor } \\
\text { de riesgo }\end{array}$ & $\begin{array}{c}\text { Método } \\
\text { diagnóstico }\end{array}$ & $\begin{array}{l}\text { Demora en } \\
\text { el diagnóstico }\end{array}$ & $\begin{array}{c}\text { Seno } \\
\text { afectado }\end{array}$ & Tratamiento & $\begin{array}{c}\text { Escala } \\
\text { funcional }\end{array}$ \\
\hline 1 & 5 años & M & $\begin{array}{l}\text { Cefaleas, vómitos, } \\
\text { irritabilidad }\end{array}$ & $\begin{array}{l}\text { Meduloblastoma, } \\
\text { anemia }\end{array}$ & RM & 48 horas & SVTD & HBPM & 0 \\
\hline 2 & 4 días & M & Crisis neonatales & Infección connatal & $\mathrm{RM}$ & 24 horas & SVTI & No & 3 \\
\hline 3 & $\begin{array}{l}4 \text { años } \\
\text { y } 1 \text { mes }\end{array}$ & M & $\begin{array}{l}\text { Cefalea, VI par, } \\
\text { somnolencia }\end{array}$ & $\begin{array}{l}\text { Factor } V \text { de Leyden, } \\
\text { OMA, OI, anemia }\end{array}$ & $\mathrm{RM}$ & 24 horas & SVTI & HBPM & 0 \\
\hline 4 & 5 años & $\mathrm{F}$ & $\begin{array}{l}\text { Cefalea/vómitos, VI par, } \\
\text { diplopía, papiledema }\end{array}$ & $\begin{array}{l}\text { Otomast. } \\
\text { izquierda }\end{array}$ & $\mathrm{RM}$ & 4 días & SVSI & HBPM & 0 \\
\hline 5 & 18 horas & M & Crisis neonatales & Distocia G20210A & $\mathrm{TAC}$ & 24 horas & SVLS, SR, SVTI & No & 2 \\
\hline 6 & 17 años & M & $\begin{array}{l}\text { Cefalea, vómitos, } \\
\text { depresión concien. }\end{array}$ & Neumonía & TAC & 24 horas & SVLSP + hem. OI & No & 2 \\
\hline 7 & $\begin{array}{l}2 \text { años y } \\
3 \text { meses }\end{array}$ & $\mathrm{F}$ & Crisis convulsivas & $\begin{array}{l}\text { Otomast. izquierda, } \\
\text { sepsis, anemia }\end{array}$ & TAC & 24 horas & SVSI & HBPM & 0 \\
\hline 8 & 1 mes y 11 días & $\mathrm{F}$ & Crisis convulsivas & Sepsis, anemia & $\mathrm{RM}$ & 24 horas & STD, HSE, isq. T-OI & No & 0 \\
\hline 9 & $\begin{array}{l}3 \text { años y } \\
9 \text { meses }\end{array}$ & M & $\begin{array}{l}\text { Vómitos, cefaleas, } \\
\text { mareos, irritabilidad }\end{array}$ & $\begin{array}{l}\text { Otomast. izquierda, } \\
\text { anemia }\end{array}$ & TAC & 72 horas & SVSI, SVTI & HBPM & 0 \\
\hline 10 & 20 horas & M & Crisis neonatales & $\begin{array}{l}\text { Sepsis tardía, } \\
\text { anemia }\end{array}$ & Angio-RM & 15 días & $\begin{array}{l}\text { SVLS, SVTI, } \\
\text { hem. F-P }\end{array}$ & No & 3 \\
\hline 11 & 10 años & M & Crisis convulsivas & $\begin{array}{l}\text { Linfoma, quimioterapia, } \\
\text { deshidratación, anemia }\end{array}$ & TAC & 48 horas & SVLS & HBPM & 0 \\
\hline 12 & 5 meses & $\mathrm{F}$ & $\begin{array}{c}\text { Crisis convulsivas, } \\
\text { hemiparesia derecha }\end{array}$ & $\begin{array}{l}\text { Shock séptico, } \\
\text { anemia }\end{array}$ & $\mathrm{RM}$ & 10 días & $\begin{array}{l}\text { SVTD + isq. FD, } \\
\text { restos } h .\end{array}$ & No & 4 \\
\hline 13 & $\begin{array}{l}3 \text { años y } 6 \text { meses } \\
\text { (Chediak-Higashi) }\end{array}$ & $\mathrm{F}$ & Crisis convulsivas & $\begin{array}{l}\text { Mastoiditis, sepsis, } \\
\text { shock, anemia }\end{array}$ & $\mathrm{RM}$ & 48 horas & SVTD & No & $\begin{array}{l}2 \text { (muerte } \\
\text { por SHF) }\end{array}$ \\
\hline 14 & 2 horas & M & Crisis neonatales & $\begin{array}{l}\text { Parto distócico, } \\
\text { anemia }\end{array}$ & RM & 24 horas & $\begin{array}{l}\text { SVLS + HSD } \\
\text { T-P derecho, } \\
\text { HSA mínima }\end{array}$ & HBPM & 0 \\
\hline 15 & 1 año y 8 meses & M & Somnolencia, vómitos & Neumonía, anemia & TAC & 18 horas & $S V L S+S T$ & HBPM & 0 \\
\hline 16 & $\begin{array}{c}1 \text { año y } \\
10 \text { meses }\end{array}$ & M & Crisis convulsivas & $\begin{array}{l}\text { Meningitis, } \\
\text { shock séptico }\end{array}$ & RM & 48 horas & $\begin{array}{c}\text { SVTI + isq. F-P izq. } \\
+ \text { hemorragia } \\
\text { occipital }\end{array}$ & HBPM & 0 \\
\hline 17 & 10 meses & M & Crisis convulsivas & Meningitis, anemia & TAC & 24 horas & SVTI & HBPM & 0 \\
\hline 18 & 3 años & M & $\begin{array}{c}\text { Cefaleas, vómitos, } \\
\text { somnolencia, papiledema }\end{array}$ & Neumonía & TAC & 6 horas & $\begin{array}{l}\text { SVLS + SR, SVT } \\
\text { bilateral, SVSI }\end{array}$ & HBPM & 1 \\
\hline 19 & 1 año & $M$ & $\begin{array}{l}\text { Cefaleas, vómitos, } \\
\text { III y V pares craneales }\end{array}$ & $\begin{array}{l}\text { Otomast., meningitis, } \\
\text { anemia }\end{array}$ & $\mathrm{RM}$ & 24 horas & SVCI & HBPM & 0 \\
\hline 20 & 6 años & $\mathrm{F}$ & $\begin{array}{l}\text { Irritabilidad alterna, } \\
\text { depresión concien., cefaleas }\end{array}$ & $\begin{array}{l}\text { Mastoiditis, } \\
\text { meningitis }\end{array}$ & $\mathrm{RM}$ & 72 horas & SVTD & HBPM & 0 \\
\hline 21 & $\begin{array}{l}5 \text { meses } \\
\text { y } 26 \text { días }\end{array}$ & M & $\begin{array}{l}\text { Crisis convulsivas, } \\
\text { irritabilidad, vómitos }\end{array}$ & $\begin{array}{l}\text { Meningococcemia, } \\
\text { anemia }\end{array}$ & $\mathrm{RM}$ & 8 días & SVTD & No & 0 \\
\hline
\end{tabular}


Tabla. Muestra de los pacientes incluidos en el trabajo (cont.).

\begin{tabular}{|c|c|c|c|c|c|c|c|c|c|}
\hline $\begin{array}{l}\text { Paciente } \\
\text { n.․ }\end{array}$ & Edad & Sexo & $\begin{array}{l}\text { Presentación } \\
\quad \text { clínica }\end{array}$ & $\begin{array}{c}\text { Factor } \\
\text { de riesgo }\end{array}$ & $\begin{array}{l}\text { Método } \\
\text { diagnóstico }\end{array}$ & $\begin{array}{l}\text { Demora en } \\
\text { el diagnóstico }\end{array}$ & $\begin{array}{l}\text { Seno } \\
\text { afectado }\end{array}$ & Tratamiento & $\begin{array}{l}\text { Escala } \\
\text { funcional }\end{array}$ \\
\hline 22 & 7 días & M & Crisis neonatales & Sin factor evidente & TAC & 5 horas & SVLSP & HBPM & 0 \\
\hline 23 & 5 años & $\mathrm{F}$ & Crisis convulsivas & $\begin{array}{l}\text { LLA en tratamiento de } \\
\text { quimioterapia, factor V } \\
\text { de Leyden, anemia }\end{array}$ & TAC & 24 horas & $\begin{array}{c}\text { SVTI + SVSI, } \\
\text { hemorragia } \\
\text { occipital izquierda }\end{array}$ & HBPM & 1 \\
\hline 25 & 1 año y 1 mes & $\mathrm{F}$ & Irritabilidad, vómitos & OMA izquierda & RM & 12 días & SVTD & HBPM & 0 \\
\hline 26 & 24 horas & $\mathrm{F}$ & Crisis neonatales & Parto distócico & $\mathrm{RM}$ & 24 horas & $\begin{array}{c}\text { SVTI + HSD } \\
\text { y tent. bilateral }\end{array}$ & No & 5 \\
\hline 29 & 10 años & $\mathrm{F}$ & Crisis convulsivas & $\begin{array}{c}\text { Otomast. bilateral, } \\
\text { homocigoto, MTHFR, } \\
\text { anemia }\end{array}$ & TAC & 24 horas & SVSD & No & 2 \\
\hline 30 & 6 años & M & $\begin{array}{c}\text { Cefaleas, parálisis VI par, } \\
\text { papiledema bilateral }\end{array}$ & Otomast. izquierda & RM & 72 horas & SVTI & HBPM & 0 \\
\hline
\end{tabular}

HBPM: heparinas de bajo peso molecular; hem. F-P: hemorragia frontoparietal; hem. OI: hemorragia occipital izquierda; HSA: hemorragia subaracnoidea; HSD: hematoma subdural derecho; HSD T-P: hematoma subdural temporoparietal; HSE: hematoma subdural espontáneo; Isq FD: isquemia frontal derecha; Isq T-OI: isquemia temporooccipital izquierda; restos h: restos hemáticos; LLA: leucemia linfoide aguda; MTHFR: metil-tetrahidrofolato-reductasa; OMA: otitis media aguda; RM: resonancia magnética; SR: seno recto; SVLS: seno venoso longitudinal superior; SVLSP: seno venoso longitudinal superior posterior; SVS: seno venoso sigmoides; SVTD: seno venoso transverso derecho; SVTI: seno venoso transverso izquierdo; TAC: tomografía axial computarizada.

\section{Tratamiento}

Se optó por la anticoagulación en 21 casos (68\%) y la abstención terapéutica en 10 (32\%), sin recurrencia sintomática en el seguimiento clínico posterior ni complicaciones graves secundarias a la anticoagulación. Un paciente falleció por descompensación de su patología inmunológica de base, sin relación con la TVC. La valoración funcional realizada y los hallazgos obtenidos en el seguimiento clínico según escala modificada de Rankin se detallan en la tabla.

\section{Discusión}

Desde las primeras descripciones realizadas en 1825 , ha parecido evidente que las TVC constituyen un reto diagnóstico para el clínico que se enfrenta a ellas. Su amplia gama de manifestaciones semioló- gicas sutiles y escasamente específicas son resultado de una patogénesis compleja, en la que intervienen una combinación de factores ambientales, genéticos y adquiridos, que de una u otra forma afectan el equilibrio natural entre los sistemas protrombóticos y trombolíticos. Dicha variabilidad clínica dificulta y muchas veces retrasa el diagnóstico, con una media de demora que en nuestro trabajo se situó en 6,5 días.

La mayoría de los escenarios en los que tienen lugar puede ocurrir a cualquier edad y está supeditada, entre otras cosas, a la presencia de ciertos factores precipitantes, actuando en un contexto de riesgo y obligando al clínico a considerar el diagnóstico ante un amplio espectro de manifestaciones neurológicas, como las convulsiones, el coma, las cefaleas o el síndrome de hipertensión endocraneal $[1,2,4]$. Más aún, considerando que patologías muy prevalentes en la infancia, como las infecciones óti- 
cas, los traumatismos craneales, la meningitis, las anemias o el síndrome nefrótico, pueden verse complicadas por una TVC, resulta lógico pensar que exista una infravaloración de su real incidencia [5].

Producto de una compleja interrelación de factores intra y extravasculares inmersos en un terreno favorecedor, existe una estrecha conexión entre factores etiológicos y la edad de presentación clínica, tal como hemos podido comprobar en nuestro trabajo. Es así como en el grupo de recién nacidos, el estado hipercoagulable de la sangre materna, sumado al elevado hematocrito fetal, el lento flujo venoso y los menores niveles de inhibidores fisiológicos de la coagulación, constituyen un importante factor de riesgo de TVC. Por otro lado, el modelaje extremo que sufre la cabeza fetal en el canal de parto o la instrumentalización de partos distócicos, como en tres de los casos presentados, pueden ser una causa clara de lesión de senos durales y llevar a su trombosis.

En lactantes y niños, en cambio, numerosas condiciones pueden predisponer a una TVC, permitiendo al clínico identificar factores de riesgo en un alto porcentaje de los casos (el 97\% en nuestra serie), en ocasiones de manera combinada $[4,6]$. Desde procesos infecciosos pericraneales (celulitis, otitis, mastoiditis o sinusitis), estados de deshidratación o fallo renal por sepsis y síndromes paraneoplásicos, hasta enfermedades crónicas o sistémicas, como el síndrome nefrótico, el lupus u otras enfermedades del tejido conectivo, se han descrito clásicamente en la bibliografía [2,4,5,7]. Múltiples trastornos hematológicos se han asociado también a las trombosis cerebrales, como las deficiencias hereditarias o adquiridas de proteínas $\mathrm{C}, \mathrm{S}$ o antitrombina III, y la homocisteinemia secundaria a mutaciones del gen de la MTHFR. No obstante, se ha señalado que la presencia del factor $V$ de Leyden o la mutación del gen de la protrombina G20210A serían las anomalías genéticas de mayor impacto como marcadores de riesgo, y el síndrome antifosfolipídico, el principal factor de riesgo adquirido $[1,5,6]$. Muchos autores en el campo de la hematología, no obstante, advierten sobre el cuidado que se debe tener en asignar responsabilidades etiológicas a marcadores trombofílicos aislados [1]. En tal sentido, merece la pena señalar que, en la serie presentada, no hemos encontrado factores protrombóticos aislados, sino asociados en un contexto de riesgo (Tabla). Curiosamente, y a pesar de la poca atención que ha recibido en adultos, en niños la anemia ha sido una hallazgo constante en más del $50 \%$ de los trabajos publicados, incluido el nuestro (61\%) [2]. Resta por definir el papel de todos estos trastor- nos como factores coadyuvantes o simplemente responsables de una trombosis cerebral [5].

De acuerdo con la bibliografía, hemos encontrado un predominio del sexo masculino (Tabla) $[6,8]$. La mayoría de informes ha documentado un importante predominio de TVC en neonatos, no obstante lo cual nuestra población de estudio se distribuyó heterogéneamente en todos los rangos etarios [5]. Los síntomas más prominentes en la muestra analizada fueron las convulsiones, junto con los signos de hipertensión endocraneal (cefaleas, trastornos de conciencia, irritabilidad, vómitos, parálisis del III, IV o VI pares craneales y papiledema). Clásicamente, se ha sugerido que, en recién nacidos y lactantes, la presentación clínica está dominada por las convulsiones, a diferencia del niño mayor, que, como hemos visto, suele comenzar con cefaleas, alteración de conciencia, vómitos y papiledema de aparición subaguda $[2,8,9,10]$. Teniendo en cuenta que muchos de estos niños cumplirán con criterios diagnósticos de pseudotumor cerebri, el diagnóstico diferencial con TVC debe ser siempre un paso obligado, razón por la cual, en la experiencia de los autores, sería aconsejable la realización en estos pacientes de una RM con contraste y en fase venosa [2].

En cuanto a la localización del seno venoso comprometido, hemos encontrado similitudes con otras series en niños, y se compromete mayoritariamente el sistema venoso superficial con afectación del seno transverso, en primer lugar, seguido del longitudinal superior, el sigmoides y una combinación de todos ellos (Tabla) $[1,8,11]$. Estudios anatómicos que han realizado tinciones post mortem del sistema vascular han colaborado extensamente en el conocimiento del sistema de drenaje venoso cerebral. Gracias a ellos, se ha podido demostrar que, cuando ocurre una trombosis del seno longitudinal superior, los infartos se localizan predominantemente en la corteza cerebral o en la sustancia blanca. En cambio, si la trombosis afecta al sistema venoso profundo, los infartos suelen localizarse fundamentalmente en la región talámica y cerebelosa. Ésta es la razón por la cual, en neonatos a término, se debe sospechar una trombosis del sistema venoso profundo ante toda hemorragia talámica o intraventricular [12]. En líneas generales, en nuestra serie de niños, se pudo establecer una aceptable correlación entre el seno trombosado y la localización del daño cerebral.

Ahora bien, el avance experimentado en los últimos años en el campo de la neuroimagen ha permitido ampliar las posibilidades diagnósticas, y el clínico debe escoger, entre una serie de técnicas, la más idónea. Es así como la tomografía computari- 
Figura 1. Signo del delta lleno en la tomografía computarizada sin contraste.

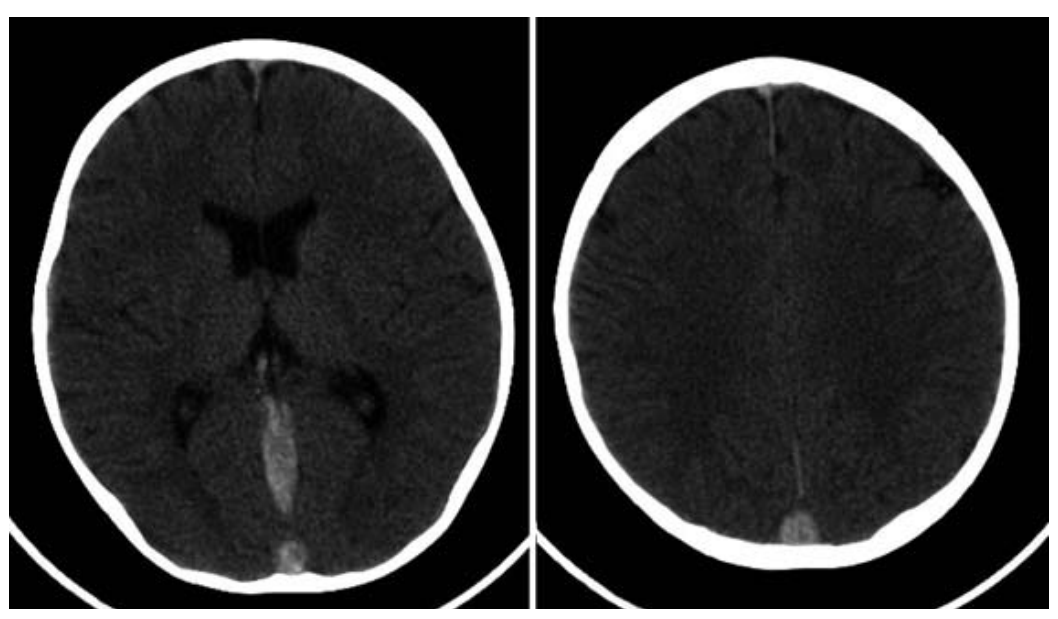

zada (TC) simple de cráneo continúa siendo el examen inicial solicitado en la mayoría de los casos. Su fácil acceso, su rapidez en pacientes muchas veces inestables y la capacidad de detectar hemorragias hacen de ella un examen frecuentemente solicitado de primera elección en urgencias. El signo diagnóstico clásico es el 'delta lleno' o 'signo de la cuerda' en la TC sin contraste y el 'delta vacío' en la TC con contraste (secundario al aumento de la densidad del trombo dentro de los senos venosos en el primero, y la ausencia de captación de contraste por defecto de llenado en el segundo) (Fig. 1). No obstante, el signo del delta vacío se encuentra presente tan sólo en el 10-20\% de pacientes, pudiendo ser la TC completamente normal en un $20 \%$ de los casos (especialmente cuando es sin contraste) o con un porcentaje no desdeñable de falsos positivos en neonatos $[4,13,14]$. Más aún, considerando que en niños, y especialmente en recién nacidos, se debe intentar evitar la irradiación ionizante de la TC, existe consenso actual en que la técnica de confirmación diagnóstica de las TVC debe ser la RM, de ser necesario con fase venosa (Fig. 2) [15]. En fase aguda (primeros cinco días) muestra la ausencia de flujo venoso en $T_{1}-T_{2}$ (trombo iso e hipointenso, respectivamente), que luego se muestra hiperintenso en ambas secuencias en la fase subaguda (5-30 días). La fase venosa, entre tanto, evidencia defectos de llenado de los senos durales principales, lográndose incluso secuencias angiográficas de la vasculatura venosa. El advenimiento de la RM por difusión-perfusión ha permitido ampliar nuestras posibilidades diagnósticas en las TVC, evidenciando un incremento en los coeficientes de difusión por edema citotóxico cuando existe un infarto venoso asociado. Otra técnica no invasiva recientemente descrita, la susceptibility weighted imaging, se ha demostrado como un método diagnóstico de gran valor al detectar precozmente diferencias de señal de sangre desoxigenada (intensidad de baja señal) capaces de delimitar tanto las diferentes estructuras venosas intracraneales normales y patológicas, como las áreas de infarto venoso con bajo tenor de oxígeno [16]. Finalmente, la angio-TC también se ha mostrado una técnica de utilidad en las TVC, reservándose los estudios angiográficos para aquellos casos en los que dichas técnicas menos invasivas no logren confirmar el diagnóstico.

El principal objetivo terapéutico de una trombosis cerebral debe estar abocado a su prevención. Se debe dirigir el máximo esfuerzo a la identificación y aplicación de medidas preventivas, tendentes a minimizar el impacto de ciertos factores predisponentes y precipitantes conocidos, que actúan sobre un terreno genético determinado. Para ello resulta de fundamental importancia instaurar oportunamente una serie de medidas sintomáticas y de soporte, con una adecuada hidratación y un manejo antibiótico racional según noción epidemiológica, sin olvidar un correcto control de crisis y de la presión intracraneal.

Sin embargo, y aunque cada vez menos, el principal punto de debate radica en el uso de la anticoagulación, especialmente en el recién nacido. Diferencias dependientes de la edad en los sistemas de coagulación y en el funcionamiento del sistema nervioso central hacen especialmente difícil extrapolar los resultados obtenidos en adultos a la población pediátrica. No obstante, en niños, la buena respuesta y tolerancia obtenida mediante su utilización hacen de la heparina (ya sea fraccionada o de bajo peso molecular) el fármaco de primera elección en los primeros siete días de tratamiento, seguido de anticoagulantes orales los restantes 3-6 meses. En tal sentido, a inicios del 2010 se presentó una extensa cohorte de 162 pacientes de entre 0 a 18 años con TVC, y se analizó la seguridad del tratamiento anticoagulante [17]. Estos autores concluyeron que la anticoagulación es segura, y que la abstención terapéutica aumenta considerablemente el riesgo de propagación del trombo y se asocia a nuevos infartos venosos, progresión de los síntomas y peor pronóstico [17]. Curiosamente, esta propagación sería silente aproximadamente en la mitad de los niños y en todos los recién nacidos incluidos en la muestra, por lo que se recomienda, en caso de 
abstención terapéutica, repetir la imagen en el día cinco [17]. En la experiencia de los autores, la tasa de complicaciones por la anticoagulación fue nula (incluso en pacientes con hemorragia intracraneal), y de los 10 pacientes de la muestra que no se anticoagularon, ocho presentaron secuelas neurológicas en diferente grado a largo plazo, no contando en ninguno de ellos con estudio de neuroimagen de control precoz.

Por otro lado, si bien el pronóstico funcional en neonatos es peor que en niños, su tasa de repermeabilización bajo tratamiento es más rápida y completa. Por esta razón, en recién nacidos y en ausencia de hemorragia significativa, se defiende el inicio precoz de anticoagulación (con heparinas de bajo peso molecular) por un mínimo de seis semanas y un máximo de tres meses, como medida para prevenir eventuales complicaciones [17-19]. En caso de hemorragia intracraneal significativa, se sugiere realizar entre el día cinco y siete una monitorización de la trombosis mediante estudios de neuroimagen. De detectarse su propagación, se sugiere el inicio de anticoagulación, aunque dicha propuesta terapéutica requiere más estudios aleatorizados que la avalen [18].

Finalmente, en nuestro actual estado de conocimiento, el uso de otras técnicas más agresivas de revascularización del seno venoso, como el tratamiento con trombolíticos, carece de suficiente evidencia científica y experiencia en niños y recién nacidos que avale su aplicación sistemática en estos casos [20]. La utilización de terapia fibrinolítica local en niños se ha descrito en casos aislados, con buen resultado en general, y se ha instaurado en casos de rápido deterioro neurológico que no responden al tratamiento anticoagulante clásico [20].

En cuanto al pronóstico a largo plazo en nuestros niños, éste fue favorable, con un porcentaje estimado de recurrencia igual al de otras series y en torno al 3\% [19]. Al igual que Kenet et al, hemos encontrado en un paciente que la oclusión persistente en la neuroimagen y la mutación en heterocigosis del gen de la protrombina G20210A resultan buenos predictores de recurrencia en las TVC, y la abstención terapéutica anticoagulante es el tercero [21].

En conclusión, las TVC constituyen una importante, pero subdiagnosticada, causa de ictus en la infancia. Ante todo paciente con clínica de hipertensión endocraneal o convulsiones en presencia de ciertos factores de riesgo conocidos, la sospecha clínica debe ser alta. Parece ser clara la seguridad y necesidad de considerar firmemente la anticoagulación en niños con trombosis venosa cerebral (grado
Figura 2. Resonancia magnética sagital ponderada en $\mathrm{T}_{1}$ en la que se observa extensa trombosis (señal hiperintensa) del seno longitudinal superior, con extensión caudal al seno recto.

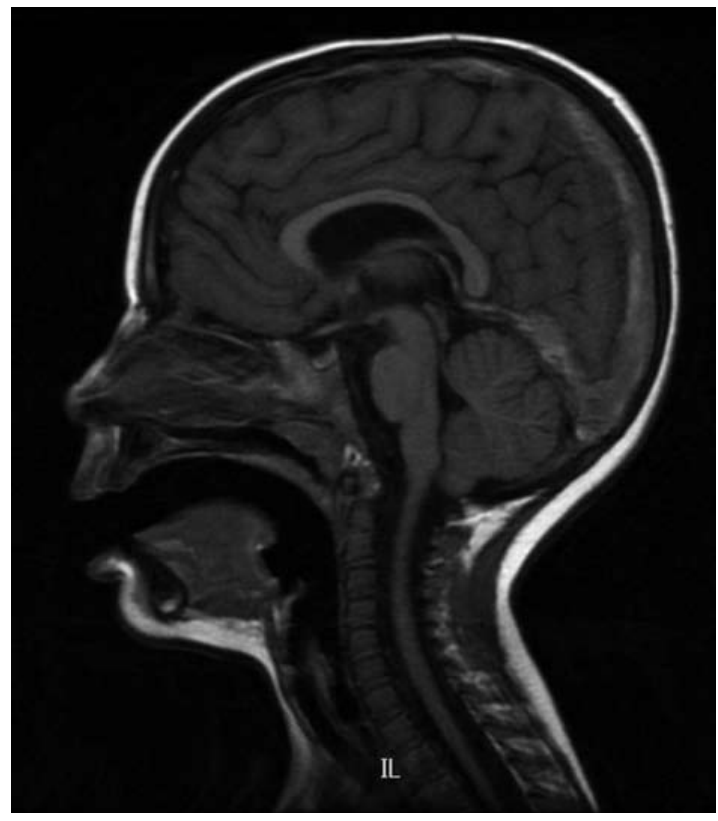

de evidencia 1B), especialmente en ausencia de hemorragia inicial [22]. En este sentido, nuestro actual estado de conocimientos nos debe obligar a centrar nuestros esfuerzos en evaluar, en un futuro cercano, la eficacia de la anticoagulación en neonatos y niños con trombosis venosas cerebrales hemorrágicas.

Bibliografía

1. Ramenghi LA, Govaert P, Fumagalli M, Bassi L, Mosca F. Neonatal cerebral sinovenous thrombosis. Semin Fetal Neonatal Med 2009; 14: 278-83.

2. Sébire G, Tabarki B, Saunders DE, Leroy I, Liesner R, Husson $\mathrm{B}$, et al. Cerebral venous sinus thrombosis in children: risk factors, presentation, diagnosis and outcome. Brain 2005; 128: 477-89.

3. Bonita R, Beaglehole R. Recovery of motor function after stroke. Stroke 1988; 12: 1497-500.

4. Cohen JE, Boitsova S, Itshayek E. Cerebral venous sinus thrombosis. Isr Med Assoc J 2009; 11: 685-8.

5. DeVeber G, Andrew M, Adams C, Bjornson B, Booth F, Buckley DJ, et al. Cerebral sinovenous thrombosis in children. N Engl J Med 2001; 345: 417-23.

6. Heller G, Heinecke A, Junker R, Knofler R, Kosch A, Kurnik K, et al. Cerebral venous thrombosis in children: a multifactorial origin. Circulation 2003; 16: 1362-7.

7. Fluss J, Geary D, DeVeber G. Cerebral sinovenous thrombosis and idiopathic nephritic syndrome in childhood: report of four new cases and review of the literature. Eur J Pediatr 2006; 165: 709-16. 
8. Vieira JP, Luis C, Monteiro JP, Temudo T, Campos MM, Quintas S, et al. Cerebral sinovenous thrombosis in children: clinical presentation and extension, localization and recanalization of thrombosis. Eur J Ped Neurol 2010; 14: 80-5.

9. Wasay M, Kojan S, Dai AI, Bobustuc G, Sheikh Z. Headache in cerebral venous thrombosis: incidence, pattern and location in 200 consecutive patients. J Headache Pain 2010; 11: 137-9.

10. Estey A, Lynch T, Lim RK. Cerebral sinovenous thrombosis masquering as migraine: a case report. Pediatr Emerg Care 2009; 25: 174-6.

11. Teksam M, Moharir M, DeVeber G, Shroff M. Frequency and topographic distribution of brain lesions in pediatric cerebral venous thrombosis. AJNR Am J Neuroradiol 2009; 29: 1961-5.

12. Shroff M, DeVeber G. Sinovenous thrombosis in children. Neuroimaging Clin N Am 2003; 13: 115-38.

13. Leach JL, Fortuna RB, Jones BV, Gaskill-Shipley MF. Imaging of cerebral venous thrombosis: current techniques, spectrum of findings, and diagnostic pitfalls. Radiographics 2006; 26 (Suppl 1): S19-41.

14. Masuhr F, Mehraein S, Einhaupl K. Cerebral venous and sinus thrombosis. J Neurol 2004: 251: 11-23.

15. Eichler F, Krishnammoorthy K, Grant PE. Magnetic resonance imaging evaluation of possible neonatal sinovenous thrombosis. Pediatr Neurol 2007; 37: 317-23.

16. Kawabori M, Kuroda S, Kudo K, Terae S, Kaneda M, Nakayama $\mathrm{N}$, et al. Susceptibility-weighted magnetic resonance imaging detects impaired cerebral hemodynamics in the superior sagittal sinus thrombosis -case report. Neurol Med Chir (Tokyo) 2009; 49: 248-51.

17. Moharir M, Shroff M, Stephens D, Pontigon AM, Chan A, MacGregor D, et al. Anticoagulants in pediatric cerebral sinovenous thrombosis: a safety and outcome study. Ann Neurol 2010; 67: 590-9.

18. Monagle P, Chalmers E, Chan A, DeVeber G, Kirkham F, Massicotte $\mathrm{P}$, et al. Antithrombotic therapy in neonates and children: American College of Chest Physicians evidence based clinical practice guidelines. Chest 2008; 23: 26-31.

19. Jordan LC, Rafay MF, Smith SE, Askalan R, Zamel KM, DeVeber G, et al. Antithrombotic treatment in neonatal cerebral sinovenous thrombosis: results of the International Pediatric Stroke Study. J Pediatr 2010; 156: 704-10.

20. Bernal-Rodríguez R, Simón de las Heras R, Mateos-Beato F, Muñoz-González A. Trombosis venosa intracraneal: a propósito de 11 casos pediátricos. Rev Neurol 2008; 46: 273-9.

21. Kenet G, Kirkham F, Niederstadt T, Heinecke A, Saunders D, Stoll M, et al. Risk factors for recurrent venous thromboembolism in the European collaborative paediatric database on cerebral venous thrombosis. A multicentre cohort study. Lancet Neurol 2007; 6: 595-603.

22. Velasco-Puyó P, Boronat-Guerrero S, Del Toro-Riera M, Vásquez-Méndez E, Roig-Quilis M. Hipertensión intracraneal asociada a trombosis de senos venosos cerebrales y mastoiditis. A propósito de dos casos pediátricos. Rev Neurol 2009; 49: 529-32.

\section{Cerebral venous thromboses in the paediatric age: clinical presentation, risk factors, diagnosis and treatment}

Introduction. Since its first description at the beginning of the 19th century, greater awareness of this disorder has been achieved, becoming nowadays a recognized but sometimes underdiagnosed cause of ischemic stroke in childhood.

Aim. To describe a population of children with cerebral venous thrombosis, analyzing clinical presentation, risk factors, treatment and evolution.

Patients and methods. A descriptive, retrospective and longitudinal review of children from 0 to 17 years of age diagnosed with sinovenous thrombosis was conducted, during the period of time between 2004 and 2009.

Results. Thirty one cases with cerebral sinus thrombosis were identified, 18 of them were boys. Clinical presentation included 18 patients with seizures and 13 with high intracranial pressure. We found infections as a risk factor in 23 patients, 3 newborns with obstetric trauma, 5 patients with prothrombotic states, 4 with chronic systemic diseases, 5 with more than one risk factor and 1 with none. Diagnosis was done by magnetic resonance imaging in 18 patients, computed tomography in 11 and magnetic resonance angiography in the other 2. Regarding location, 16 patients showed compromise of the transverse sinus, 4 of the superior longitudinal sinus, another 4 an affectation of the sigmoid sinus, 2 of the cavernous sinus and 5 with a combination of them. Anticoagulation therapy was done in 21 patients and treatment abstention in 10. None of the patients suffered complications or death due to anticoagulation therapy or sinovenous thrombosis. No recurrent symptomatic thrombosis in long term follow up was found.

Conclusions. Sinovenous cerebral thromboses are an important but underestimated cause of stroke in childhood. Clinical suspicion should be high, in patients with risk factors and intracranial hypertension or seizures.

Key words. Anticoagulation. Cerebral sinovenous thrombosis. Childhood. Ictus. Mastoiditis. Risk factors. 